\title{
Top Quark Properties Measurements with the ATLAS Detector
}

\section{Antonio Limosani*}

The University of Sydney and CERN

E-mail: antonio.limosaniesydney.edu.au

The top quark is unique among the known quarks in that it decays before it has an opportunity to form hadronic bound states. This makes measurements of its properties particularly interesting as one can access directly the properties of a bare quark. Measurements of the charge asymmetry in top quark pair production, which probe models of physics beyond the Standard Model, are presented; these include measurements at high invariant masses of the $t \bar{t}$ system using boosted top quarks. Limits on the rate of flavour-changing neutral currents in the production or decay of the top quark are discussed.

38th International Conference on High Energy Physics 3-10 August 2016

Chicago, USA

* Speaker.

${ }^{\dagger}$ On behalf of the ATLAS collaboration. 


\section{Introduction}

Ever since the top quark's existence was inferred by Kobayashi and Maskawa to explain CP violation in kaon decays it has captivated particle physicists. It is unique among the quarks in having a lifetime shorter than the time required for hadronisation. Its properties are especially sensitive to the effects of beyond Standard Model (SM) particles. Here measurements of the charge asymmetry and searches for a top quark decaying via flavour-changing neutral current decay are presented. The data corresponding to an integrated luminosity of $20.3 \mathrm{fb}^{-1}$ are collected from LHC proton collisions at energy $\sqrt{s}=8 \mathrm{TeV}$ with the ATLAS detector [1].

\section{Top quark Pair Production Charge Asymmetries}

At the LHC the asymmetry of a (anti-)top quark being favourably produced in the direction of the incoming (anti-)quark is explored through the difference in absolute rapidities, $|y|$, of top and anti-top quarks, where the charge asymmetry is defined as follows

$$
A_{C}^{t \bar{t}}=\frac{N(\Delta|y|>0)-N(\Delta|y|<0)}{N(\Delta|y|>0)+N(\Delta|y|<0)}
$$

with $\Delta|y| \equiv\left|y_{t}\right|-\left|y_{\hat{t}}\right|$. The harder spectrum of valence quarks in the proton and the correlation of the outgoing top quark direction with the direction of the incoming quark make the top quark's rapidity distribution broader than that of the anti-top quark [2]. However this asymmetry is diluted due to top quark pair production being dominated by gluon-fusion processes at the LHC, which exhibit no charge asymmetry. At next-to-leading order in QCD, $A_{C}^{t \bar{t}}=0.0111 \pm 0.0004$ [2]. An asymmetry is also present in the case of dileptons from top quark pair production, $A_{C}^{l l}$ where above $\Delta|y|$ is replaced by $\Delta|\eta| \equiv\left|\eta_{l^{+}}\right|-\left|\eta_{l^{-}}\right|$. At next-to-leading order in QCD, $A_{C}^{l l}=0.0064 \pm 0.0003$ [2].

SM QCD processes introduce a charge asymmetry at order $\alpha_{s}^{3}$ through interference between initial and final state radiation diagrams and between box and tree diagrams. Particles predicted in theories beyond the SM could provide anomalous contributions in both $s$-channel and $t$-channel top quark pair production through an axi-gluon [3] or a new heavy gauge boson [4], respectively.

The nominal $t \bar{t}$ signal sample is generated at NLO in QCD using P owHEG (version 1, r2330) [5] and the CT10 [6] parton distribution function (PDF) set, setting the $h_{\text {damp }}$ parameter to the top quark mass of $172.5 \mathrm{GeV}$. The $t \bar{t}$ cross section for $p p$ collisions at a center-of-mass energy of $8 \mathrm{TeV}$ is set to $\sigma_{t \bar{t}}=253_{-15}^{+13} \mathrm{pb}$, calculated at NNLO in QCD including resummation of next-to-next-to-leading logarithmic (NNLL) soft gluon terms with top++2.0 [7].

A measurement of the charge asymmetry in the lepton-plus-jets final state of $t \bar{t}$ production is presented. The $t \bar{t}$ pairs are selected in the single-lepton channels ( $e$ or $\mu)$ with at least four jets. A kinematic fit [8] that assesses the compatibility of the observed event with the decays of a $t \bar{t}$ pair based on a likelihood approach is used to reconstruct the event kinematics. The reconstructed inclusive distribution of $\Delta|y|$ and the distributions as a function of invariant mass, $m_{t \bar{t}}$, transverse momentum, $p_{T, t \bar{t}}$, and longitudinal boost, $\beta_{Z}$, of the $t \bar{t}$ system, were unfolded to obtain results that can be directly compared to theoretical computations. The inclusive $t \bar{t}$ charge asymmetry is measured to be $A_{C}^{t \bar{t}}=0.009 \pm 0.005$ (stat.+syst.), where the statistical uncertainty is dominant, while signal modelling is the leading systematic uncertainty. All measurements are statistically 
limited and compatible with the SM prediction within the uncertainties. The measurements in the $\ell+$ jets final state are fully described elsewhere [9]. Fig 1 shows the measurement of $A_{C}$ as a function of $m_{t \bar{t}}$ and $\beta_{z, t \bar{t}}$, where in each bin the measurement is consistent with the SM expectation as well as heavy and light color octet model predictions. The precision of the measurements also allows for the exclusion of a large phase-space of the parameters describing various beyond SM scenarios as shown in Fig. 1c.

A measurement of the rapidity-dependent charge asymmetry in top quark pair production that is based on techniques (as described in Refs. [10]) specifically designed to deal with the collimated decay topology of boosted top quarks is performed, where the top quark pair is produced with a large invariant mass. The analysis focuses on the lepton+jets $(\ell+$ jets $)$ final state, where the hadronic top quark decay is reconstructed as a single large-radius (large- $R$ ) jet and tagged as such using jet substructure variables. The leptonic top quark decay is reconstructed from a single smallradius (small- $R$ ) jet, a single charged lepton (muon or electron), and missing transverse momentum, corresponding to the neutrino from the $W$ boson decay. The charge asymmetry in a fiducial region with large invariant mass of the top quark pair $\left(m_{t \bar{t}}>0.75 \mathrm{TeV}\right)$ and an absolute rapidity difference of the top- and anti-top quark candidates within $-2<\left|y_{t}\right|-\left|y_{\hat{t}}\right|<2$ is measured to be $(4.2 \pm$ $3.2) \%$, in agreement with the SM prediction at next-to-leading order. A differential measurement in three $t \bar{t}$ mass bins is shown in Fig. 2a whilst Fig. 2b shows that combined with the forward backward asymmetry $A_{F B}$ measured at the Tevatron, $A_{C}$, is able to constrain many beyond SM physics scenarios. This measurement is described in detail elsewhere [11].

Measurements of the leptonic and $t \bar{t}$ charge asymmetry in the dilepton channel, characterised by two high- $p_{\mathrm{T}}$ leptons (electrons or muons), are presented. The reconstruction of the $t \bar{t}$ system is achieved using the kinematic (KIN) method [12], assuming values of $172.5 \mathrm{GeV}$ and $80.4 \mathrm{GeV}$ for the top quark and $W$ boson masses, respectively, which allows the system of equations that relate the particle momenta at each of the decay vertices in the process to be solved numerically by the Newton-Raphson method. Inclusive and differential measurements as a function of $m_{t \bar{t}}, p_{\mathrm{T}, \mathrm{t}}$, and $\beta_{z, t \bar{t}}$ of the $t \bar{t}$ system are performed in the full phase space as well as in a fiducial volume based on the detector acceptance and selection requirements, using particle-level objects. The measurement in the fiducial region does not rely on extrapolating to regions of phase space that are not within the detector acceptance, while the full phase space measurement has the benefit of being comparable to theoretical calculations at the parton level, including beyond SM models. Measurements are compatible with the SM and do not exclude the two sets of both light and heavy octet BSM models, where the predictions for the latter are compared with measurement in Fig 2c. The full sets of measurements are described in detail elsewhere [13].

\section{Search for Flavour-Changing Neutral Current Decays of Top quarks}

In the SM, due to the GIM mechanism [14], flavour-changing neutral current (FCNC) decays of top quarks are forbidden at tree level. They are allowed at one-loop level, but with a suppression factor of several orders of magnitude with respect to the dominant decay mode [15]. However, several SM extensions predict higher branching ratios (BRs), for example the two-Higgs-doublet model [16], and the minimal supersymmetric model (MSSM) [17]. For a complete review see Ref. [18]. 


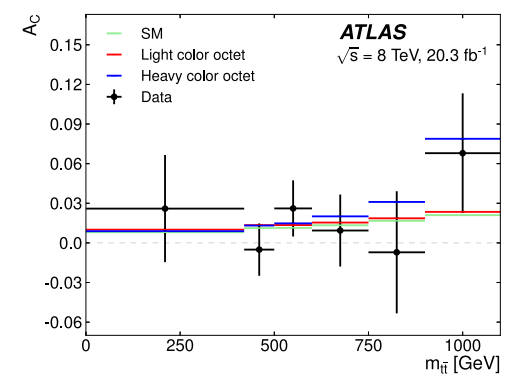

(a)

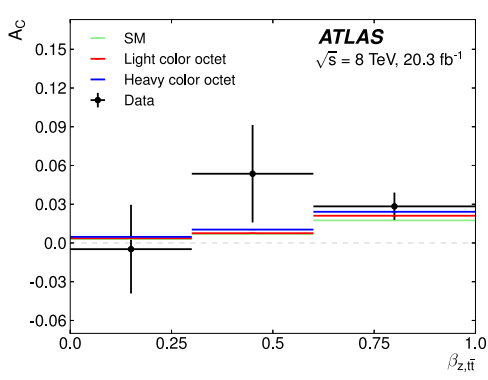

(b)

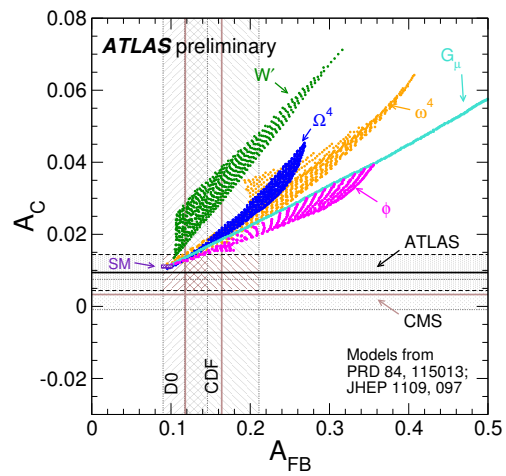

(c)

Figure 1: In the lepton-plus-jets measurement, measured $A_{C}^{t \bar{t}}$ values as a function of bin-averaged (a) $m_{t \bar{t}}$ and (b) $\beta_{z, t \bar{t}}$ compared with predictions for SM and for right-handed colour octets with masses below the $t \bar{t}$ threshold and beyond the kinematic reach of current LHC searches. (c) Measured inclusive charge asymmetries $A_{C}^{t \bar{t}}$ at the LHC versus forward-backward asymmetries $A_{\mathrm{FB}}$ at Tevatron, compared with the SM predictions as well as predictions incorporating various potential BSM contributions: a $W^{\prime}$ boson, a heavy axigluon $\left(\mathscr{G}_{\mu}\right)$, a scalar isodoublet $(\phi)$, a colour-triplet scalar $\left(\omega^{4}\right)$, and a colour-sextet scalar $\left(\Omega^{4}\right)$. The horizontal bands and lines correspond to the ATLAS and CMS measurements, while the vertical ones correspond to the CDF and D0 measurements. The uncertainty bands correspond to a $68 \%$ confidence level interval. The plots are taken from [9]. .

A search for FCNC decays of a top quark to an up-type quark $(q=u, c)$ and the SM Higgs boson, where the Higgs boson decays to $b \bar{b}$, is presented. The analysis searches for top quark pair events in which one top quark decays to $W b$, with the $W$ boson decaying leptonically, and the other top quark decays to $\mathrm{Hq}$. Data are analysed in the lepton-plus-jets final state, characterised by an isolated electron or muon and at least four jets. The search exploits the high multiplicity of $b$ quark jets characteristic of signal events, and employs a likelihood discriminant that uses the kinematic differences between the signal and the background, which is dominated by $t \bar{t} \rightarrow W b W b$ decays. No significant excess of events above the background expectation is found, and observed (expected) 95\% CL upper limits of $0.56 \%$ (0.42\%) and $0.61 \%(0.64 \%)$ are derived for the $t \rightarrow H c$ $(t \rightarrow H u)$ branching ratios respectively. The combination of this search with other ATLAS searches in the $H \rightarrow \gamma \gamma$ and $H \rightarrow W W^{*}, H \rightarrow \tau \tau$ decay modes significantly improves the sensitivity, yielding observed (expected) 95\% CL upper limits on the $t \rightarrow H c(t \rightarrow H u)$ branching ratios of $0.46 \%$ $(0.25 \%)$ and $0.45 \%(0.29 \%)$ respectively. The measurement is described in detail elsewhere [19].

A search for the FCNC decay $t \rightarrow q Z$ is presented. Top quark pair-production events with one top quark decaying through the $t \rightarrow q Z(q=u, c)$ channel and the other through the dominant SM mode $t \rightarrow b W$ are considered as signal. Only the decays of the $Z$ boson to charged leptons and leptonic $W$ boson decays are used. The reconstructed objects include 3 isolated electrons or muons, at least 2 jets, where one or two of these jets should be b-tagged; and some missing transverse energy. Control regions are used to assign background modelling uncertainties, which are the dominant systematics. After selection the expected number of background events is $4.7 \pm 0.4 \pm 1.0$ (dominant backgrounds include $W Z(1.3), t \bar{t} V(1.4)$ and $t Z(1.0)$ ), and 3 events are observed in data. No evidence for a signal is found and an observed (expected) upper limit on the $t \rightarrow q Z$ branching 


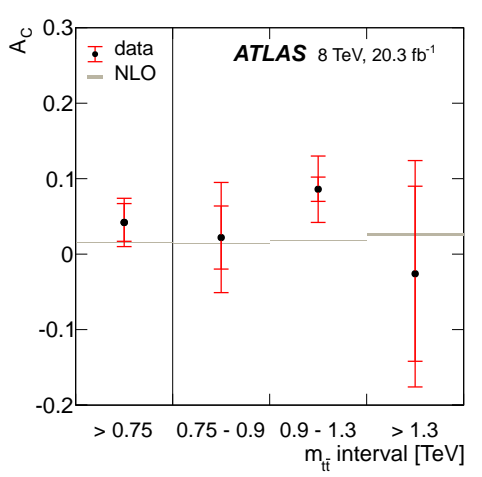

(a)

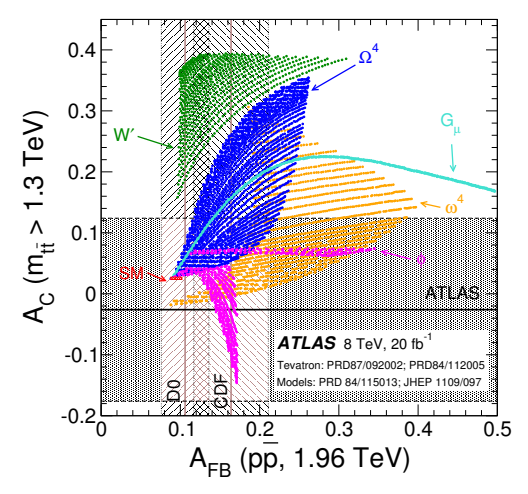

(b)

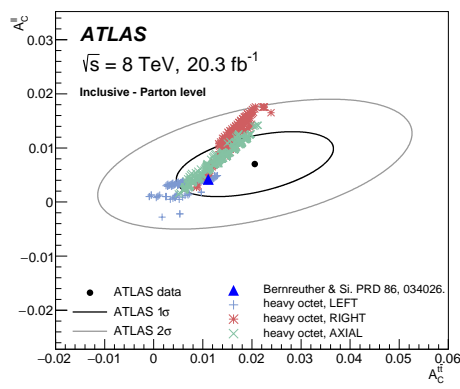

(c)

Figure 2: (a) In the boosted lepton-plus-jets measurement a summary of the charge asymmetry measurements, where the error bars on the data indicate the modelling and unfolding systematic uncertainties, shown as the inner bar, and the total uncertainty, which includes the statistical uncertainty and the experimental systematic uncertainties. The SM prediction of the NLO calculation in Ref. [2] for the charge asymmetry of top quark pairs with $|\Delta| y||<2$ is indicated as a shaded horizontal bar in each $m_{t \bar{t}}$ bin, where the width of the bar indicates the uncertainty. (b) Predictions from a number of extensions of the SM for the forward-backward asymmetry integrated over $m_{t \bar{t}}$ at the Tevatron (on the $x$-axis in both plots) and two high-mass charge asymmetry measurements at the LHC. The $y$-axis in both figures represents the measurement for $m_{t \bar{t}}>1.3 \mathrm{TeV}$. The SM predictions of both the forward-backward asymmetry at the Tevatron and the charge asymmetry at the LHC are also shown. (c) In the dilepton final state a comparison of the inclusive $A_{\mathrm{C}}^{\ell \ell}$ and $A_{\mathrm{C}}^{t \bar{t}}$ measurement values in the full phase space to the SM NLO QCD+EW prediction and to a benchmark BSM model with a heavy octet with mass beyond the reach of the LHC, for various couplings as described in the legend. Ellipses corresponding to $1 \sigma$ and $2 \sigma$ combined statistical and systematic uncertainties of the measurement, including the correlation between $A_{\mathrm{C}}^{\ell \ell}$ and $A_{\mathrm{C}}^{t \bar{t}}$, are also shown. (a) and (b) are taken from Ref. [11] and (c) is taken from [13].

ratio of $7 \times 10^{-4}\left(8 \times 10^{-4}\right)$ is set at the $95 \%$ confidence level. The measurement is described in detail elsewhere [20].

\section{Conclusion}

ATLAS charge asymmetry measurements are found to be consistent with the SM, and thereby rule out parameter space in many BSM scenarios. ATLAS FCNC top quark decays are providing improved upper limits and beginning to reach the sensitivity capable of ruling out beyond SM scenarios.

\section{References}

[1] ATLAS Collaboration, JINST 3, S08003 (2008).

[2] J. H. Kuhn and G. Rodrigo, JHEP 1201, 063 (2012)

W. Bernreuther and Z. G. Si, Phys. Rev. D 86, 034026 (2012)

[3] A. R. Zerwekh, Phys. Lett. B 704, 62 (2011) 
[4] J. Shelton and K. M. Zurek, Phys. Rev. D 83, 091701 (2011)

[5] S. Alioli, P. Nason, C. Oleari and E. Re, JHEP 1006, 043 (2010)

S. Frixione, P. Nason and C. Oleari, JHEP 0711, 070 (2007)

P. Nason, JHEP 0411, 040 (2004)

[6] H. L. Lai, M. Guzzi, J. Huston, Z. Li, P. M. Nadolsky, J. Pumplin and C.-P. Yuan, Phys. Rev. D 82, 074024 (2010)

[7] M. Cacciari, M. Czakon, M. Mangano, A. Mitov and P. Nason, Phys. Lett. B 710, 612 (2012)

M. Beneke, P. Falgari, S. Klein and C. Schwinn, Nucl. Phys. B 855, 695 (2012)

P. Baernreuther, M. Czakon and A. Mitov, arXiv:1206.0621 [hep-ph].

M. Czakon and A. Mitov, JHEP 1212, 054 (2012)

M. Czakon and A. Mitov, JHEP 1301, 080 (2013)

M. Czakon, P. Fiedler and A. Mitov, Phys. Rev. Lett. 110, 252004 (2013)

M. Czakon and A. Mitov, Comput. Phys. Commun. 185, 2930 (2014)

[8] J. Erdmann, S. Guindon, K. Kroeninger, B. Lemmer, O. Nackenhorst, A. Quadt and P. Stolte, Nucl. Instrum. Meth. A 748, 18 (2014)

[9] ATLAS Collaboration, Eur. Phys. J. C 76, no. 2, 87 (2016)

[10] ATLAS Collaboration, JHEP 1508 (2015) 148

ATLAS Collaboration, Phys. Rev. D 88, no. 1, 012004 (2013)

ATLAS Collaboration, JHEP 1209, 041 (2012)

ATL-PHYS-PUB-2010-008

[11] ATLAS Collaboration, Phys. Lett. B 756, 52 (2016) doi:10.1016/j.physletb.2016.02.055 [arXiv:1512.06092 [hep-ex]].

[12] A. Abulencia et al. [CDF Collaboration], Phys. Rev. D 73, 112006 (2006)

T. Aaltonen et al. [CDF Collaboration], Phys. Lett. B 722, 48 (2013)

[13] ATLAS Collaboration, Phys. Rev. D 94, no. 3, 032006 (2016)

[14] S. L. Glashow, J. Iliopoulos and L. Maiani, Phys. Rev. D 2, 1285 (1970).

[15] J. A. Aguilar-Saavedra, Acta Phys. Polon. B 35, 2695 (2004)

[16] D. Atwood, L. Reina and A. Soni, Phys. Rev. D 55, 3156 (1997)

[17] J. J. Cao, G. Eilam, M. Frank, K. Hikasa, G. L. Liu, I. Turan and J. M. Yang, Phys. Rev. D 75, 075021 (2007)

[18] K. Agashe et al. [Top Quark Working Group Collaboration], arXiv:1311.2028 [hep-ph].

[19] ATLAS Collaboration, Eur. Phys. J. C 76, no. 2, 55 (2016)

[20] ATLAS Collaboration, Eur. Phys. J. C 76, no. 1, 12 (2016) 